\title{
Changes in gustatory function in postmenopausal women
}

\section{Evaluation of gustatory function in postmenopausal women \\ C. Delilbasi, T. Cehiz, U. K. Akal and T. Yilmaz Br Dent J 2003; 194: 447-449}

Objective

The aim of this study is to evaluate the gustatory function in postmenopausal women.

Subjects and methods

Whole mouth taste test and spatial taste test were administered to 20 postmenopausal women (Group 1) and 20 age-matched male subjects (Group 2). Further investigations were carried out for subjective assessment of taste alteration and nutritional differences between premenopausal and postmenopausal periods.

\section{Results}

There was a significant reduction in sucrose perception and in palatal sensitivity in postmenopausal women $(p<0.05)$. Only 7 (35\%) of the female patients noticed alteration in taste perception during the postmenopausal period. Alteration in dietary habits after menopause was reported by $9(45 \%)$ patients.

\section{Conclusion}

This study demonstrates that taste perception of the tongue does not change, but that there is a disorder in palatal perception in postmenopausal women. Furthermore, there is tendency to preference of sweeter foods during menopause.

\section{IN BRIEF}

- The taste perception of the tongue does not change, but there is a decline in palatal perception in postmenopausal women.

- Postmenopausal women appear to have a reduced perception of sucrose.

- This can alter eating habits, such as preference for sweeter foods.

- The dental practitioner plays a key role in the management of the oral complications of the menopause.

\section{COMMENT}

Menopause is a normal developmental stage in a woman's life after permanent cessation of menstruation. Menopause is accompanied by a number of characteristic changes; some of which occur in the oral cavity. It has been shown that estrogen can affect many oral tissues such as salivary glands, temporomandibular joints, oral mucosa and jawbones. Changes in the function of taste buds and neural networks have been noted to occur following menopause. This effect on taste and neuronal function causes alterations in taste perception. Alteration in taste function is induced by reduction in saliva production, dysesthesia and atrophic gingivitis. Furthermore the gustatory sense is further complicated by osteoporosis of the jaws, dental caries and periodontitis.

In this research, the authors have shown anatomical localization of taste alteration in postmenopausal women. This opens the need for further investigations to better understand the mechanism of oral sensory disturbance. Postmenopausal women are more prone to certain diseases parallel to growing age, and change in hormonal status. The finding that indicates reduction in sucrose perception and therefore preference of sweeter food during menopause proves valuable information about the relation between dietary habits and general medical status of the patients.

Women have unique oral health needs, which change throughout their lives. The results of this study provide practical knowledge for dental practitioners who are responsible for managing oral complications of menopause.

Mikihiko Kogo

Professor and Head, Osaka University Graduate School of Dentistry, First Department of Oral and Maxillofacial Surgery 